\title{
First results of auroral tomography from ALIS-Japan multi-station observations in March, 1995
}

\author{
Takehiko Aso $^{1}$, Masaki Ejiri ${ }^{1}$, Akira Urashima ${ }^{2}$, Hiroshi Miyaoka ${ }^{1}, \AA_{k}$ ke Steen ${ }^{3}$, Urban Brändström ${ }^{3}$, and Björn Gustavsson ${ }^{3}$ \\ ${ }^{1}$ National Institute of Polar Research, Itabashi, Tokyo 173, Japan \\ ${ }^{2}$ Department of Electrical Engineering, Kyoto University, Kyoto 606-01, Japan \\ ${ }^{3}$ Swedish Institute of Space Physics, P.O. Box 812, S-981 28 Kiruna, Sweden
}

(Received March 26, 1997; Revised September 10, 1997; Accepted October 6, 1997)

\begin{abstract}
Auroral tomography observations have been carried out in March, 1995, as a joint international campaign between Sweden and Japan. Three unmanned Swedish ALIS stations (Kiruna, Merasjärvi, Tjautjas) and two Japanese JICCD sites (Abisko, Nikkaluokta), geographically separated by about $50 \mathrm{~km}$ at higher latitudes, were operated to capture multi-station monochromatic tomography images at $557.7 \mathrm{~nm}$ wavelength using CCD cameras. All cameras were pointing to one of the predetermined directions to secure a common field of view. Several images of auroral arcs, mostly for the core region right above Kiruna, have synchronously been taken by the multi-station imaging system. Tomographic inversion analysis for four-point images was carried out using the algebraic reconstruction technique. Reconstructions of a curved arc and of a double arc system suggest promising application of this technique to the retrieval of three-dimensional auroral luminosity.
\end{abstract}

\section{Introduction}

Auroral tomography is a fairly new technique to retrieve the three-dimensional (3D) luminous structure of aurora based on multiple auroral images taken simultaneously from a number of stations. This is indeed a promising application of computerized tomography (CT) used in clinical medicine to solar-terrestrial physics, and also a reasonable extension of the reconstruction of aurora forms based on binocular stereo images as discussed, for example, by Aso et al. (1990, 1993, 1994) and Frey et al. (1996). Aurora stereoscopic reconstruction stems from both altitude determination by triangulation (Stenbaek-Nielsen and Hallinan, 1979) and reconstruction of a luminous distribution by a scanning photometer chain in the magnetic meridian plane (Romick and Belon, 1967; Vallance Jones et al., 1991). Determination of monochromatic 3D structures and simultaneous satellite data on the impinging particle energy together with the model atmosphere will help to quantify how the particles interact with the earth atmosphere to excite luminous auroras.

For years, the optical group at the Swedish Institute of Space Physics has been constructing the ALIS (an acronym for Auroral Large Imaging System), which is a large network of ground-based aurora imaging stations designed to realize high spatial and temporal resolution (Steen and Brändström, 1993). The network has also the potential for tomographic imaging by pointing the cameras to particular regions at auroral heights. Fourteen stations are the goal of the ALIS project, and at the time of the first campaign in March, 1995, three stations were completed. Integration of two manned JICCD (short for Japanese Intensified CCD) cameras into this network thus yielded five stations. In this paper, some

Copy right (C) The Society of Geomagnetism and Earth, Planetary and Space Sciences (SGEPSS); The Seismological Society of Japan; The Volcanological Society of Japan; The Geodetic Society of Japan; The Japanese Society for Planetary Sciences. new results for the tomographic reconstruction of stable auroral arcs are presented based on four-station images. The aurora here is located less favorably, viz, slightly north relative to the magnetic zenith of observing stations. The results, however, suggest the capability of auroral tomography in retrieving luminous structure from a limited number of stations by adding some constraints to this underdetermined inverse problem.

Numerical simulations are carried out both for the feasibility tests of reconstruction and for the evaluation of analyzed structures. The result gives a measure of retrieval or more specifically its uniqueness for the number and relative locations of observing sites for the model aurora which is based on the actually observed aurora.

\section{Observation}

The first multi-static optical tomographic observation of aurora employed three remotely-controlled ALIS stations (Kiruna, Merasjärvi, Tjautjas) and two manned Japanese sites (Abisko and Nikkaluokta), as shown in Fig. 1. Each ALIS camera uses a high sensitivity quad cooled CCD detector with quantum efficiency greater than $80 \%$ and low read-out noise, and a telecentric lens is installed between the wide-angle lens and the interference filter. A 16 bits/pixel image field of $1024 \times 1024$ pixels comprises four quadrants to reduce frame read-out time by parallel processing. The JICCD is a CCD TV camera with an interference filter and an image intensifier. A composite video output is averaged by a hardware image processor and fed to a personal computer through an 8 bits/pixel image capture board with 512 $\times 512$ resolution. These images are stored in the FITS (Flexible Image Transport System) format.

Monochromatic $557.7 \mathrm{~nm}$ images were taken for the exposure (on-chip integration for ALIS) or average (over NTSC frames for JICCD) time of 1 3 seconds when an auroral arc was coming up to a pre-determined common 


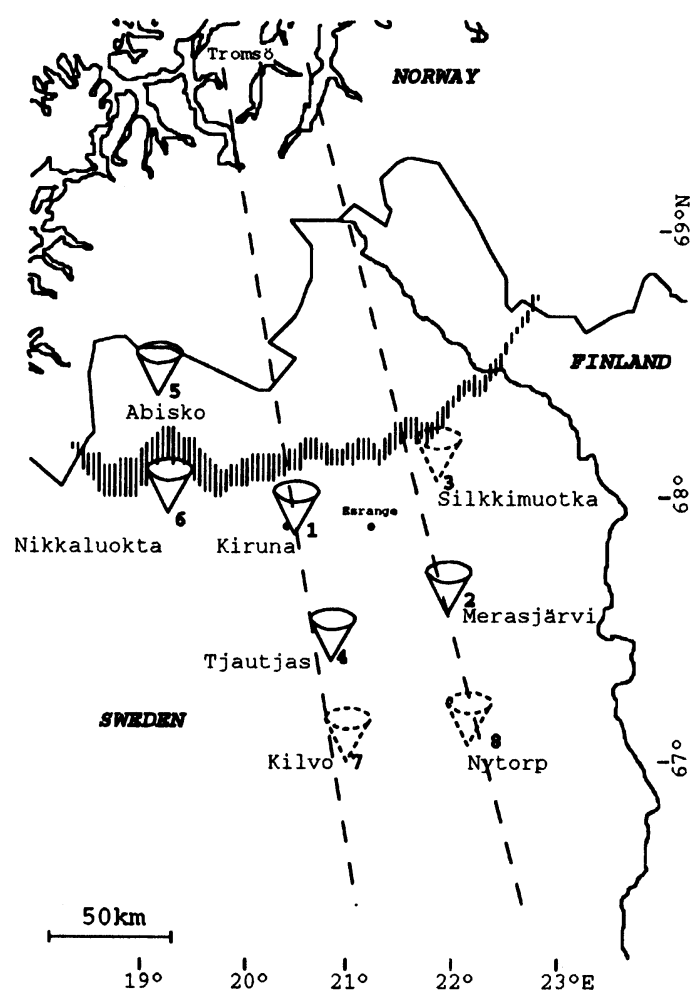

Fig. 1. A network for the auroral tomographic observation.

field-of-view to which all cameras were pointing. We actually had four regions, core region overhead of Kiruna, regions south and north of Kiruna, and one farther north (over EISCAT at Tromsö) regions. Present analyses pertain only to the core region. For unmanned ALIS operations, camera pointing, image taking and house keeping commands are all given from the control center (CC) in Kiruna. Exposures are synchronized to UTC by GPS receivers and all quick-look images from the five stations are transferred back to $\mathrm{CC}$ by a computer networking to monitor if identical auroral portions are in sight. During four weeks of observations, tomographic observations were made only for a couple of nights at three or four stations at most, due to cloud cover at some of the stations. In this paper, two CT images taken at four stations, Kiruna, Merasjärvi, Tjautjas and Nikkaluokta will be analysed.

\section{Tomographic Analysis}

Calibration of orientation, corrections for optical characteristics for each camera, and estimation of relative sensitivity between cameras should be made as a prerequisite to the analysis. Camera calibration uses background star images and the relationship between a pixel coordinate and the direction of corresponding line-of-sight is given for each camera and pointing direction. A lens characteristic of $2 \sin (\theta / 2)$, where $\theta$ is the angle relative to the optical axis, holds for JICCD cameras, while $\theta+2 \tan \theta$ is used for the ALIS cameras. Perspective projection planes for the optical axis direction which is estimated by the non-linear least squares using Levenberg-Marquardt method are mapped to image coordinates through a polynomial approximation (Aso et al., 1990). The results indicate fields of view for the
JICCD and ALIS cameras are about $90^{\circ}$ and $60^{\circ}$, respectively. Lens characteristics are also important to retrieve corrected gray levels for limb darkening. ALIS images were corrected for this darkening using flat field images. For the Nikkaluokta images, a correction was made based on approximate calibration images using a phosphor calibrator. Also, the calibrator was used for all cameras to measure the relative sensitivities. Atmospheric extinction is assumed constant and not explicitly taken into account in the present analysis. ALIS camera is temperature-controlled, while JICCD sensitivity also depends on high tension voltage and also change in ambient temperature.

Aurora imaging is expressed assuming a gray level $g_{i}$ for a pixel $i$ is expressed as

$$
g_{i}=\sum_{j} w_{i j} L_{j}
$$

where $w_{i j}$ means how the luminosity $L_{j}$ of a voxel (volume cell) $j$ contributes to $g_{i}$ and depends on the distance squared and the volume of each voxel contained in the conic region spanned by a pixel $i$.

The tomographic reconstruction has been based on a modification of the MART (Multiplicative Algebraic Reconstruction Technique) method (Gordon et al., 1970; Raymund et al., 1990; Gustavsson, 1992), assuming cubic voxels in the luminous region. In particular, our method is the modified version of the SIRT (Simultaneous Iterative Reconstruction Technique) (Gilbert et al., 1972) in which voxel values are only revised by taking all projection values pertinent to each voxel simultaneously as

$$
L_{j}^{(k+1)}=L_{j}^{(k)} \prod_{i}\left(\frac{g_{i}}{\tilde{g}_{i}^{(k)}}\right)^{\lambda \cdot w_{i j} / \sum_{i} w_{i j}}
$$

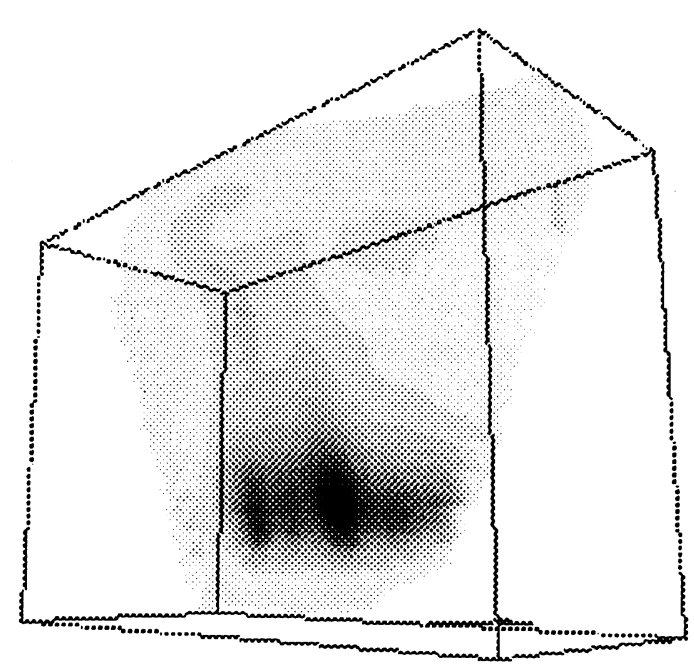

\section{$(315,80) 300300$}

Fig. 2. A tomographic reconstruction of auroral arc with a fold observed at 19:09:30 UT, March 26, 1995, viewed from southeast with zenith angle of 80 degrees. A box is 80 to $220 \mathrm{~km}$ in altitude with $90 \mathrm{~km}$ in NS and $200 \mathrm{~km}$ in EW directions. 
where $\lambda$ is a relaxation parameter $(0.5 \sim 1.4$, a typical value is 0.9 ) and $k$ is the iteration number. Equation (2) indicates each voxel value is augmented multiplicatively with the averaged weight if the observed value $g_{i}$ is greater than the reconstructed gray level $\tilde{g}_{i}^{(k)}$ and vice versa. Iterative revision of voxel values proceeds until $\tilde{g}_{i}^{(k)}$ is close to $g_{i}$ in a least squares sense. Iterations are typically $60 \sim 70$ for the present analysis.

Corresponding pixel areas in the different images used for reconstruction are selected by the epipolar line constraint. If epipolar lines in a pair of images do not traverse the whole bright region in one image, these lines are not sampled so as not to include possible contributions from an occluded region in the other image. Sampled points are some $1.5 \times 10^{5}$ over 4 images in the present analysis. An user interface has been developed in which all tomographic images are shown and, on specifying a pixel point in one image, epipolar lines are drawn in the rest of images using camera orientation calibration data. Relevant points for the common field of view are then sampled with each gray level value for use in the reconstruction.

\section{Results and Discussions}

\subsection{Aurora at 19:09:30 UT}

Four CT images at 19:09:30 UT on March 26, 1995, are analyzed by the modified SIRT method. The Merasjärvi camera was pointing to EISCAT but luminous regions to be analysed was also in sight. Voxels are assumed in this region from 80 to $220 \mathrm{~km}$ in altitude, $90 \mathrm{~km}$ in NS and $200 \mathrm{~km}$ in EW directions with voxel numbers of $70 \times 45 \times 50,2 \mathrm{~km}$ in altitude and thickness and $4 \mathrm{~km}$ in extended direction. Relative sensitivity in gray level is varied as a parameter so that residuals $\tilde{g}_{i}-g_{i}$ are minimized. This is required as the JICCD cameras are not fully calibrated for temperature fluctuations. (a)

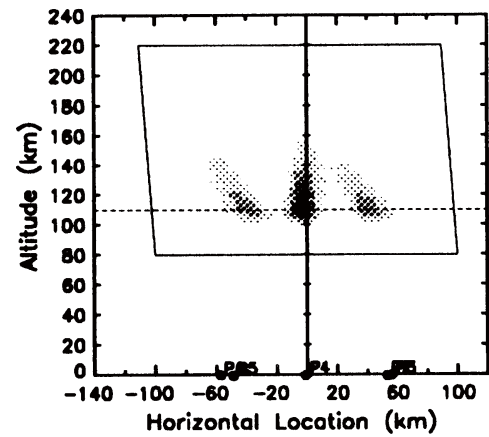

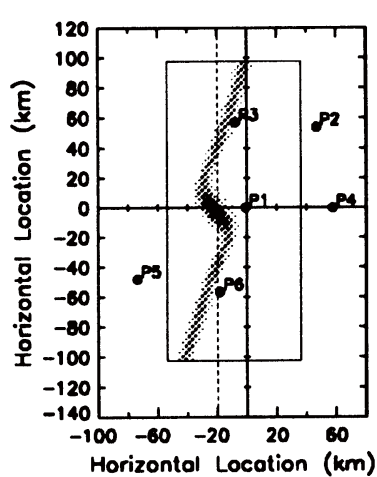

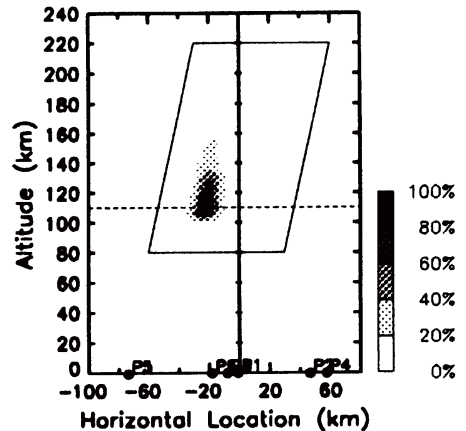

(b)

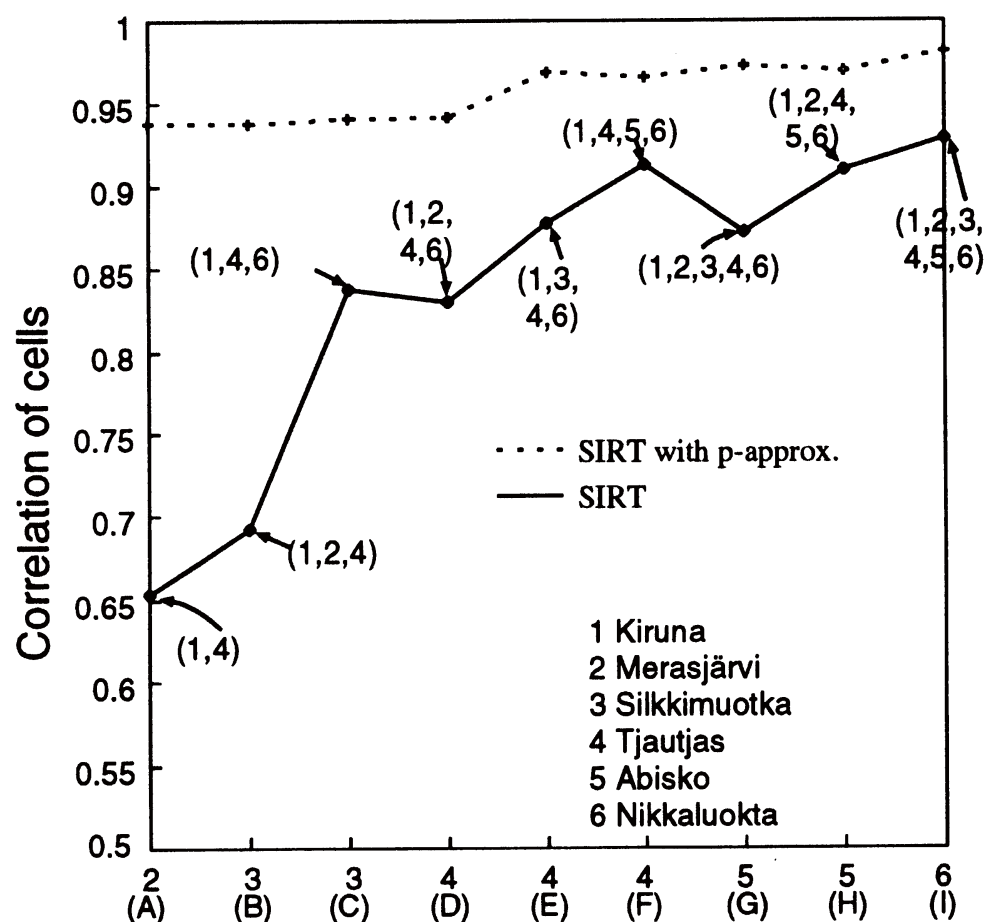

\section{Number of points}

Fig. 3. (a) An aurora model with a fold illustrated by three sectional contours. (b) Cross-correlation of reconstructed and modelled luminosity voxels versus number and combinations of sites for SIRT reconstruction with (a broken line) and without (a solid line) a proximity constraint. 


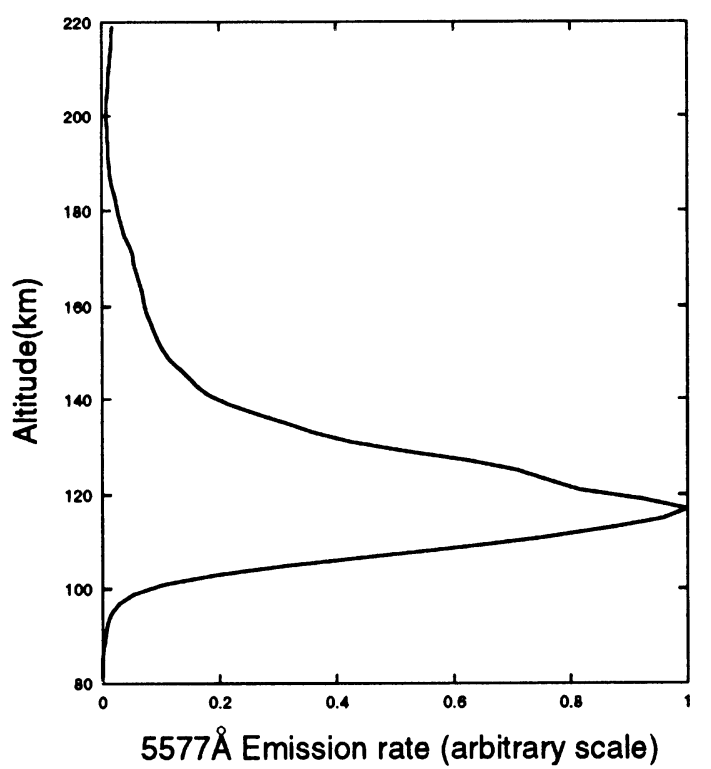

Fig. 4. Reconstructed altitude profile of auroral luminosity at 19:09:30 UT, March 26, 1995.
In these images, luminous region is fairly localized relative to the field of view of the cameras, and pixel points sampled by epipolar constraints cover almost the whole region. In this reconstruction, uniform initial guess is given to all voxels. However aurora is located slightly north of observing sites. Here, the so-called "field-alignedness correction in proximity" is assumed in which altitude profiles along the geomagnetic line of force for the near-by \pm 8 voxels in NS and \pm 4 voxels in $\mathrm{EW}$ are assumed identical and voxel values are redistributed along each line of force, scaling it to the integrated values along the line of force. This proximity-constraint is formulated as

$$
\begin{aligned}
& \tilde{L}^{(k+1)}\left(x^{\prime}, y^{\prime}, z^{\prime}\right) \\
& =\frac{\int \tilde{L}^{(k)}\left(x^{\prime}, y^{\prime}, z^{\prime}\right) d z^{\prime} \iint \tilde{L}^{(k)}\left(x^{\prime}-u, y^{\prime}-v, z^{\prime}\right) d u d v}{\iiint \tilde{L}^{(k)}\left(x^{\prime}-u, y^{\prime}-v, z^{\prime}\right) d u d v d z^{\prime}} .
\end{aligned}
$$

Here, $x^{\prime}, y^{\prime}, z^{\prime}$ is a coordinate system along the geomagnetic line of force with $z^{\prime}$ taken along it and horizontal $\left(x^{\prime}, y^{\prime}\right)$ has the same value for each geomagnetic line. In other words, this postulates that the auroral formation is basically along
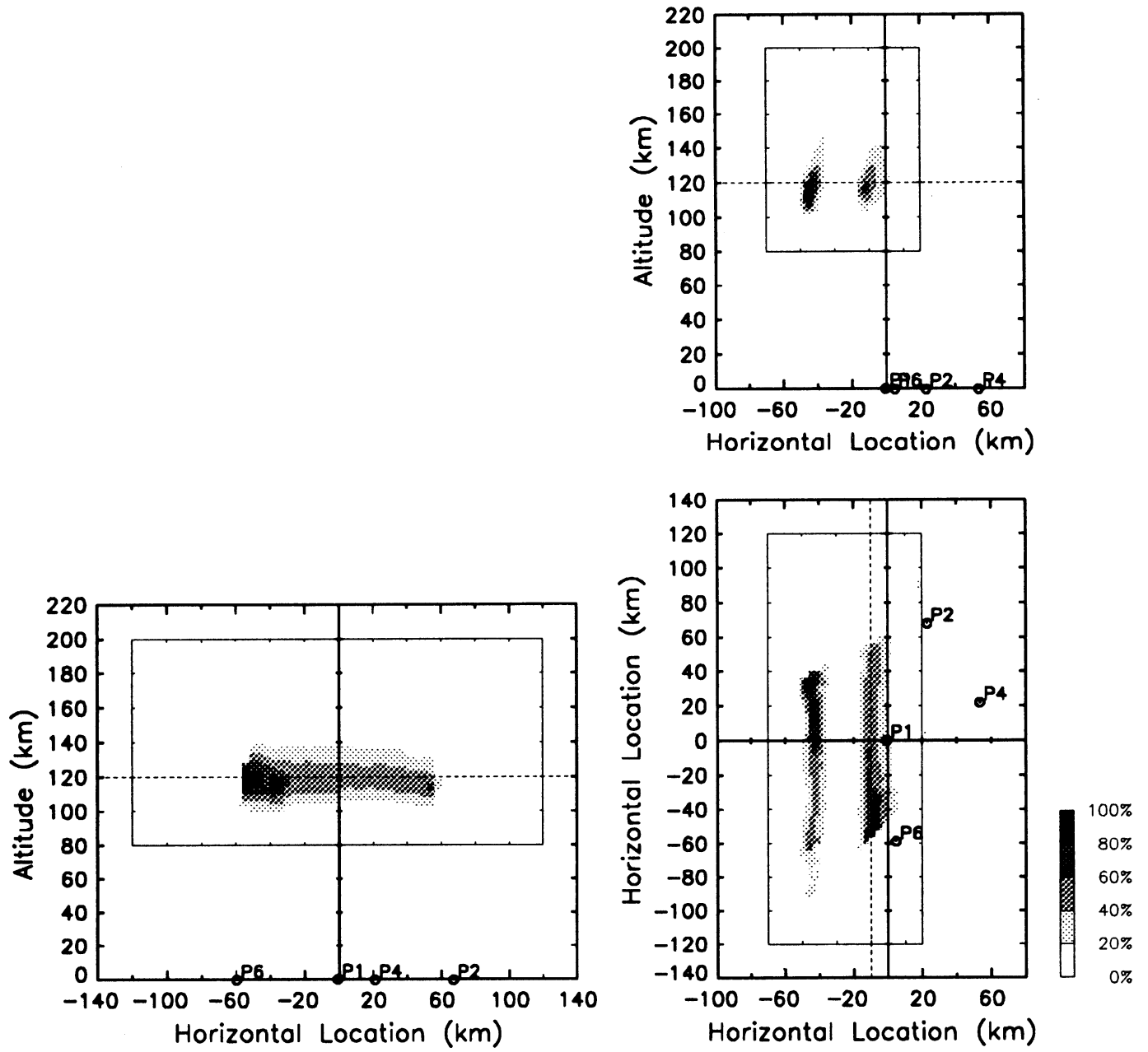

Fig. 5. A tomographic reconstruction of double arc system observed at 20:26:10 UT, March 26, 1995. Positive direction of horizontal axis in the top and bottom right panel is southward and that of the bottom left panel is eastward. The inner frame indicates the extent of voxels for the reconstruction calculation. The sites, $P_{1}, P_{2}, P_{4}, P_{6}$ refer to Kiruna, Merasjärvi, Tjautjas and Nikkaluokta, respectively. 
the geomagnetic line of force, and altitude profiles are uniform within its proximity. This adds apriori information to sometimes underdetermined auroral tomography. The SIRT iteration is then resumed, and the sequence is repeated until the residual becomes a minimum.

Figure 2 shows a visualization of the reconstructed aurora as seen from southwest with a zenith angle of $80^{\circ}$. It is seen that the auroral curtain is folding with enhanced luminosity in the middle. The altitude of this enhanced region is around $120 \mathrm{~km}$ and east-west extension of the whole reconstructed region is about $100 \mathrm{~km}$. The retrieved thickness in the almost meridional plane is about $10 \sim 15 \mathrm{~km}$ and reconstruction starting from initial values based on thinly-assumed structure, e.g., e-fold value of 5 or $8 \mathrm{~km}$ in the Gaussian profile, is not compatible with the image seen from Nikkaluokta.

A numerical test by using aurora model similar to the reconstructed structure supports the reconstruction of relatively thick region in the folded arc with intensity-reduced thin structure at both ends of the curtain. Also, the reliability of inferred structures has been checked by the reconstruction simulation using the same model as shown in Fig. 3. In the figure, the cross-correlation of model and reconstructed voxel values versus available observing sites is shown with (a broken line) and without(a solid line) a proximity constraint. It is seen that the reconstruction is by and large better for larger number of sites. Evidently, the station 5, north of the arc, plays a dominant contribution and even a 5-point observation, e.g., $(1,2,3,4,6)$ gives less reliable result than the 4-point one $(1,4,5,6)$ which includes the station 5 . Slight degradation in $(1,2,4,6)$ relative to $(1,4,6)$, in $(1,2$, $3,4,6)$ relative to $(1,3,4,6)$ or in $(1,2,4,5,6)$ relative to $(1,4,5,6)$ is due to the increase of reconstructed volume which is only in sight from the stations 1 and 2, and to passable reconstruction in this region. Also, the proximity constraint is found to be very efficient even for the 2-point observation so long as the aurora conforms to the assumption involved in the constraint.

Figure 4 shows a reconstructed altitude profile of auroral luminosity in the midst of a folded arc. It is seen that, though the quenching below the peak at around $120 \mathrm{~km}$ is less rapid, the inferred result compares reasonably with theoretical emission profile at $557.7 \mathrm{~nm}$ by monoenergetic electrons of some keV impinging on atomic oxygen (Banks and Chappel, 1974).

\subsection{Aurora at 20:26:10 UT}

Four images of a double-arc system were observed at 20:26:10 UT on the same day. When the luminous region is large compared with the common viewing area and some part is not visible from all cameras, it is in principle difficult to reconstruct 3-D structures of that part. Also, double arcs appear at farther north of the observing sites. Hence, in this analysis, the initial guess is also assumed as an additional constraint which is taken from the result obtained by the 2D SIRT analysis with uniform initial guess and proximity field-aligned constraint in the vertical plane including KirunaTjautjas pair. This helps to get rid of inevitable artifacts both high up and lower height regions in 3-D reconstruction. Voxel numbers are $60 \times 45 \times 60$ with the same voxel size as in Fig. 2, and proximity field-aligned constraint is given for $\pm 6 \times 8$ voxels in the NS and EW directions.
East-west elongated double arcs are retrieved as shown in Fig. 5. These figures indicate shaded contours in the meridional (top right) and zonal planes(bottom left) and horizontal plan (bottom right), broken lines showing the position of the cross-section for the other contours. Though assumptions in the initial guess are needed to obtain reasonable solution, a structure thus reconstructed is best consistent with information available from tomographic images and basic nature of field-alignedness of aurora. The observation of the passage of the aurora arc across the magnetic zenith as in Borovsky (1993) has been carried out simultaneously at Abisko by using a very narrow field-of-view camera, but no data was available due to cloud cover.

\section{Conclusion}

Novel application of computerized tomography to reconstruct auroral luminous structures using multiple images has been worked out. It is envisaged that multiple image information along with field aligned characteristics can give acceptable aurora structure although some constraint including initial guess is required when large-scale aurora spreads out relative to the observing network. This method will be developed by using more observing sites and other integrated data by scanning photometers and imaging riometers and preferably even the platform onboard space vehicles. Further study on the reconstruction algorithm for the increased number of sites will reconcile unconstrained analytical methods such as the filtered backprojection or convolution integral to aurora tomography in which fieldaligned constraint still works as a substantial knowledge for the reconstruction. If the analysis is made in collaboration with concomitant radar, ground-based and satellite observations, the reconstructed structure will contribute to the comprehensive study on the formation and dynamics of aurora based on the relevant atmospheric, plasma and field parameters and energy distribution of incoming auroral particles.

Acknowledgments. The authors gratefully acknowledge supports by the Grant-in-Aid for Scientific Research (No. 07640588) from the Ministry of Education, Science, Sports and Culture, Japan and by the Research Grant-in-aid for FY 1994 from the Tateisi Science and Technology Foundation. They would also appreciate the help in computational works by Messrs. Lars Nilsson and Atsufumi Fujita. Part of the computation has been carried out at the Integrated Media Environment Experimental Laboratory, Kyoto University.

\section{References}

Aso, T., T. Hashimoto, M. Abe, T. Ono, and M. Ejiri, On the analysis of aurora stereo observations, J. Geomag. Geoelectr., 42(5), 579-595, 1990.

Aso, T., M. Ejiri, H. Miyaoka, T. Ono, T. Hashimoto, T. Yabu, and M. Abe, Aurora stereo observation in Iceland, Proc. NIPR Symposium on Upper Atmos. Phys., 6, 1-14, 1993.

Aso, T., M. Ejiri, H. Miyaoka, T. Ono, Y. Tetsuro, K. Muguruma, T. Hashimoto, and M. Abe, Aurora stereo observation in Iceland and its tomography analysis, Trans. Inst. Electronics Communication Engineers, D-II, J77-D-II(1), 69-78, 1994 (in Japanese).

Banks, P. M. and C. R. Chappel, A new model for the interaction of auroral electrons with the atmosphere: Spectral degradation, backscatter, optical emission, and ionization, J. Geophys. Res., 79(10), 1459-1470, 1974. 
Borovsky, J. E., Auroral arc thickness as predicted by various theories, $J$. Geophys. Res., 98(A4), 6101-6138, 1993.

Frey, S., H. U. Frey, D. J. Carr, Os. H. Bauer, and G. Haerendel, Auroral emission profiles extracted from three-dimensionally reconstructed arcs, J. Geophys. Res., 101(A10), 21731-21741, 1996.

Gilbert, P., Iterative methods for the three-dimensional reconstruction of an object from projections, J. Theor. Boil., 36, 105-117, 1972.

Gordon, R., R. Bender, and G. T. Herman, Algebraic reconstruction technique (ART) for three-dimensional electron microscopy and X-ray photography, J. Theor. Biol., 29, 471-481, 1970.

Gustavsson, B., A study of feasible tomographic inversion techniques for ALIS, IRF Technical Report 039, 37 pp., Swedish Institute of Space Physics, Kiruna, Sweden, 1992.

Raymund, T. D., J. R. Austen, S. J. Franke, C. H. Liu, J. A. Klobuchar, and J. Stalker, Application of computerized tomography to the investigation of ionospheric structures, Radio Sci., 25(5), 771-789, 1990.

Romick, G. J. and A. E. Belon, The spatial variation of auroral luminosityII, Planet. Space Sci., 15, 1695-1716, 1967.
Steen, ̊. and U. Brändström, ALIS-A multi-station ground-based imaging system at high latitudes, STEP International Newsletter, 3(5), 11-14, 1993.

Stenbaek-Nielsen, H. C. and T. J. Hallinan, Pulsating auroras: Evidence for non-collisional thermalization of precipitating electrons, J. Geophys. Res., 84(A7), 3257-3271, 1979.

Vallance Jones, A., R. L. Gattinger, F. Creutzberg, F. R. Harris, A. G. McNamara, A. W. Yau, E. J. Llewellyn, D. Lummerzheim, M. H. Rees, I. C. McDade, and J. Margot, The ARIES auroral modelling campaign: Characterization and modelling of an evening auroral arc observed from a rocket and a ground-based line of meridian scanners, Planet. Space Sci., 39(12), 1677-1705, 1991.

T. Aso (e-mail: aso@nipr.ac.jp), M. Ejiri (e-mail: ejiri@nipr.ac.jp), A. Urashima, H. Miyaoka, A. Steen (e-mail: steen@irf.se), U. Brändström, and B. Gustavsson 